\title{
Cylindrical vector beam multiplexer/demultiplexer using off-axis polarization control
}

\author{
Shuqing Chen ${ }^{1}$, Zhiqiang Xie ${ }^{1}$, Huapeng Ye ${ }^{2}$, Xinrou Wang ${ }^{1}$, Zhenghao Guo², Yanliang He ${ }^{1}$, Ying Li ${ }^{1 凶}$, \\ Xiaocong Yuan $\mathbb{1}^{1 凶}$ and Dianyuan Fan ${ }^{1}$
}

\begin{abstract}
The emergence of cylindrical vector beam (CVB) multiplexing has opened new avenues for high-capacity optical communication. Although several configurations have been developed to couple/separate CVBs, the CVB multiplexer/ demultiplexer remains elusive due to lack of effective off-axis polarization control technologies. Here we report a straightforward approach to realize off-axis polarization control for CVB multiplexing/demultiplexing based on a metal-dielectric-metal metasurface. We show that the left- and right-handed circularly polarized (LHCP/RHCP) components of CVBs are independently modulated via spin-to-orbit interactions by the properly designed metasurface, and then simultaneously multiplexed and demultiplexed due to the reversibility of light path and the conservation of vector mode. We also show that the proposed multiplexers/demultiplexers are broadband (from 1310 to $1625 \mathrm{~nm}$ ) and compatible with wavelength-division-multiplexing. As a proof of concept, we successfully demonstrate a four-channel CVB multiplexing communication, combining wavelength-division-multiplexing and polarization-division-multiplexing with a transmission rate of $1.56 \mathrm{Tbit} / \mathrm{s}$ and a bit-error-rate of $10^{-6}$ at the receive power of $-21.6 \mathrm{dBm}$. This study paves the way for CVB multiplexing/demultiplexing and may benefit high-capacity CVB communication.
\end{abstract}

\section{Introduction}

Multiplexing, which coaxially transmits multiple signal channels, has a significant importance for increasing the optical communication density ${ }^{1-4}$. Driven by the wavelength-division-multiplexing, the transmission rate of optical communication has been incredibly increased to $\mathrm{Tbit} / \mathrm{s}^{5-7}$. However, further enhancement of the transmission rate is facing limited availability in bandwidth if only relying on adding spectrum multiplexed channels. Beyond using more spectra, the two orthogonal polarization states have also been explored for multiplexing ${ }^{8-10}$.

\footnotetext{
Correspondence: Ying Li (queenly@szu.edu.cn) or Xiaocong Yuan (xcyuan@szu. edu.cn)

${ }^{1}$ Institute of Microscale Optoelectronics, Shenzhen University, 518060 Shenzhen, China

${ }^{2}$ Guangdong Provincial Key Laboratory of Optical Information Materials and Technology \& Institute of Electronic Paper Displays, South China Academy of Advanced Optoelectronics, South China Normal University, 510006 Guangzhou, China

These authors contributed equally: Shuqing Chen, Zhiqiang Xie, Huapeng Ye
}

So far, the development of high-speed optical communication is hindered by the lack of suitable multiplexing dimensions. Recently, cylindrical vector beam (CVB) multiplexing has emerged as a powerful technique to boost signal channels ${ }^{11-17}$. The CVBs enable robust transmission ability in atmospheric turbulence due to its inherent spatially inhomogeneous polarization distribution and ability to transmit over ultra-long distance because the vector mode is the eigenmodes of few-mode fiber ${ }^{18-22}$. More importantly, CVB multiplexing is highly promising for optical communication compatible with conventional wavelength-division-multiplexing and polarization-division-multiplexing as it is independent of wavelength and polarization states.

Coupling and separating CVBs are two pivotal elements in CVB multiplexing communication. Although a large variety of devices have been proposed to modulate homogeneous light beams ${ }^{23-27}$, such as Q-plate, spatial light modulator (SLM), and spiral phase plate, the

\section{(c) The Author(s) 2021}

(c) (i) Open Access This article is licensed under a Creative Commons Attribution 4.0 International License, which permits use, sharing, adaptation, distribution and reproduction cc) in any medium or format, as long as you give appropriate credit to the original author(s) and the source, provide a link to the Creative Commons license, and indicate if changes were made. The images or other third party material in this article are included in the article's Creative Commons license, unless indicated otherwise in a credit line to the material. If material is not included in the article's Creative Commons license and your intended use is not permitted by statutory regulation or exceeds the permitted use, you will need to obtain permission directly from the copyright holder. To view a copy of this license, visit http://creativecommons.org/licenses/by/4.0/. 
modulation of light beams with spatially inhomogeneous polarization distribution remains challenging. So far, the spin-dependent Pancharatnam-Berry (P-B) phase devices have been explored to separate $\mathrm{CVBs}^{28,29}$. The mechanism is to independently control the wavefront of the respective left- and right-handed circularly polarized (LHCP/RHCP) components as CVB can be theoretically decomposed into two vortex beams with opposite-handed circular polarization and conjugate topological charges. However, although this approach can linearly separate the CVBs, the coordinate transformation destroys the polarization structure of the CVB, rendering it only effective for CVB demultiplexing ${ }^{30-32}$. Alternatively, off-axis control technologies such as miniature Dammann vortex gratings have also been investigated to couple and separate light beams ${ }^{33}$. However, it is usually limited to light beams with homogeneous polarization due to its phase-only grating structure. For CVBs with inhomogeneous polarization, a gradient phase device is required to create a gradient phase difference between the LHCP and RHCP components, so that the CVBs with off-axis incident angles can coaxially propagate and carry different phase structures. Despite considerable efforts of off-axis control technologies $^{34-36}$, the off-axis polarization control of CVBs still remains a challenge.

In this work, we report a straightforward approach to realize off-axis spin-independent polarization control for CVB multiplexing/demultiplexing based on a plasmonic metasurface. By combining the P-B phase with the propagation phase ${ }^{37-42}$, we show that off-axis control of polarization is realized with metal-dielectric-metal metasurface, which is consisting of subwavelength $\mathrm{Au}$ nanoantenna on a $\mathrm{SiO}_{2}-\mathrm{Au}-\mathrm{Si}$ substrate. In principle, the linearly polarized Gaussian beams with different incident angles are first transferred into coaxially transmitted CVBs, subsequently reflected, and then spatially separated by the metasurface. Hence, the CVB with inverse polarization order is recovered to the fundamental mode for demultiplexing. We also show that the multiplexers/demultiplexers are broadband (working wavelength ranging from 1310 to $1625 \mathrm{~nm}$ ) and compatible with wavelength-divisionmultiplexing. Furthermore, as a proof of concept, we successfully demonstrate a four-channel CVB multiplexing communication with four vector mode channels $(m= \pm 1$, \pm 2 ), combining wavelength-division-multiplexing and polarization-division-multiplexing with a transmission rate of $1.56 \mathrm{Tbit} / \mathrm{s}$ and a bit-error-rate (BER) of $10^{-6}$ at the received power of $-21.6 \mathrm{dBm}$.

\section{Results}

Principle of off-axis polarization control

$\mathrm{CVB}$, which possesses spatially inhomogeneous polarization distribution, is the axially symmetric solution to the full vector electromagnetic wave equation ${ }^{43-45}$.
Due to the polarization singularity of CVB, it inherently has a null field in the beam center. The Jones matrix of the CVB with $m$-th polarization order can be defined by:

$$
\begin{aligned}
E_{\mathrm{vector}} & =E_{0}\left[\begin{array}{c}
\cos \left(m \theta+\varphi_{0}\right) \\
\sin \left(m \theta+\varphi_{0}\right)
\end{array}\right] \\
& =E_{0}\left[\begin{array}{c}
\frac{1}{2} \exp \left[i\left(m \theta+\varphi_{0}\right)\right]+\frac{1}{2} \exp \left[-i\left(m \theta+\varphi_{0}\right)\right] \\
\frac{1}{2} \exp \left[i\left(m \theta+\varphi_{0}\right)\right]-\frac{1}{2} \exp \left[-i\left(m \theta+\varphi_{0}\right)\right]
\end{array}\right] \\
& =\frac{1}{2} E_{0} \exp \left[i\left(m \theta+\varphi_{0}\right)\right]\left[\begin{array}{c}
1 \\
-i
\end{array}\right]+\frac{1}{2} E_{0} \exp \left[-i\left(m \theta+\varphi_{0}\right)\right]\left[\begin{array}{c}
1 \\
i
\end{array}\right]
\end{aligned}
$$

where $E_{0}$ is the simplified amplitude, $m$ is the polarization order, $\theta$ is the azimuthal angle, and $\varphi_{0}$ is the initial phase. From Eq. (1), it can be seen that CVB can be considered as the linear superposition of RHCP vortex beam with helical phase of $\exp (\operatorname{im} \theta)$ and LHCP vortex beam with helical phase of $\exp (-i m \theta)$. More specifically, the two vortex beams have opposite topological charges and orthogonal circular polarizations. For example, the CVB $(m=+2)$ is the linear superposition of a RHCP vortex beam with the topological charge of +2 and a LHCP vortex beam with the topological charge of -2 . To realize off-axis control of CVB, two different grating phases corresponding to the RHCP and LHCP components are demanded. As shown in Fig. 1b, c, for the LHCP component, the transmission function can be expressed as:

$$
\Phi_{\mathrm{LHCP}}=\sum_{n=-\frac{N}{2}}^{n=\frac{N}{2}} C_{n} \exp \left[i n\left(\frac{2 \pi x}{T}+\Delta m \theta\right)\right]\left[\begin{array}{c}
1 \\
-i
\end{array}\right]
$$

where $\Delta m$ is the interval of topological charges, $T$ is the period of grating, $n$ is the diffraction order from $-N / 2$ to

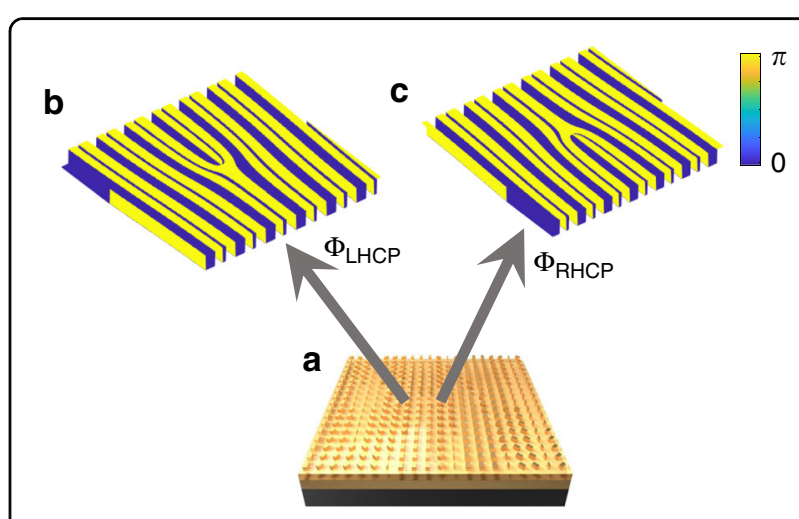

Fig. 1 Schematic of off-axis polarization control based on spinindependent phase modulation. a Device for off-axis polarization control. b, c Phase response of LHCP and RHCP components 
$N / 2$, and $\left|C_{n}\right|^{2}=1 / N$ is the power of the $n$-th order normalized with reference to the total power. Similarly, for the RHCP component, it can be expressed as:

$$
\Phi_{\mathrm{RHCP}}=\sum_{n=-\frac{N}{2}}^{n=\frac{N}{2}} C_{n} \exp \left[i n\left(\frac{2 \pi x}{T}-\Delta m \theta\right)\right]\left[\begin{array}{l}
1 \\
i
\end{array}\right]
$$

If a linearly polarized Gaussian beam is incident, the transmission function of off-axis polarization control can be described as:

$$
\Phi_{\mathrm{LP}}=\sum_{n=-\frac{N}{2}}^{n=\frac{N}{2}} C_{n} \exp \left(i n \frac{2 \pi x}{T}\right)\left[\begin{array}{l}
\cos ((n \times \Delta m) \theta) \\
\sin ((n \times \Delta m) \theta)
\end{array}\right]
$$

In principle, to achieve off-axis polarization control, the LHCP(RHCP) component should be first converted into RHCP(LHCP) by using spin-dependent phase device and then independently modulated without conjugacy. Here it should be pointed out that the polarization control cannot be accomplished because the phase differences of the conjugate phase of the binary grating are the same (see Supplementary Note 2).

\section{Realization of off-axis polarization control based on compound-phase metasurface}

The working principle of metasurface-based CVB multiplexer/demultiplexer is schematically illustrated in Fig. 2. To uniformly distribute light intensity in the far-field, we adopt the binarized Dammann vortex grating phase, where the position and number of phase-jump points in the normalized period are determined by the diffraction orders (see Supplementary Note 1). The grating periods $(T)$ are the same between RHCP and LHCP phase response, but the topological charge intervals are different, which are shown in Fig. 2a. The transmission function of LHCP and RHCP are $\Phi_{\mathrm{LHCP}}=\sum_{n=-2}^{n=2} C_{n} \exp \left[\operatorname{in}\left(\frac{2 \pi x}{T}+\theta\right)\right]$ and $\Phi_{\mathrm{RHCP}}=\sum_{n=-2}^{n=2} C_{n} \exp \left[i n\left(\frac{2 \pi x}{T}-\theta\right)\right]$, respectively. The far-field intensity distributions of different polarized incident light beams are shown in Fig. 2b. If a LHCP Gaussian beam is incident on the metasurface, four RHCP vortex beams can be obtained at the diffraction orders with topological charges of $l=-2,-1,+1,+2$, respectively. If a RHCP Gaussian beam is incident, the beams at these diffraction orders will be replaced by LHCP vortex beams with $l=+2,+1,-1,-2$. Hence, if a linearly polarized Gaussian beam (contain both LHCP and RHCP components) is incident, it leads to four CVBs with orders of $m=-2,-1,+1,+2$. However, traditional P-B phase elements perform the transformation of $|L\rangle \rightarrow \mathrm{e}^{i 2 \theta}|R\rangle$ and
$|R\rangle \rightarrow \mathrm{e}^{-i 2 \theta}|L\rangle$, where $\theta$ is the half-wave $(\pi)$ retardance rotated angles, and the LHCP/RHCP components are converted to carry opposite spin state and conjugate phase. Hence, P-B phase elements cannot independently modulate the LHCP and RHCP components, which means that vortex beam with an opposite topological charge at the same diffraction order cannot be achieved. To solve this problem, we propose to independently modulate the LHCP and RHCP components by combining the propagation phase and P-B phase (see Supplementary Note 3). Since the spin transformation from LHCP to RHCP is still needed, we employ the unit structure with half-wave plate effect $\left(\varphi_{x}-\varphi_{y}=\pi\right.$, where $\varphi_{x}$ and $\varphi_{y}$ are the propagation phase on the $x$ - and $y$-direction linear polarization, respectively). To achieve $|L\rangle \rightarrow \mathrm{e}^{i \phi_{1}}|R\rangle$ and $|R\rangle \rightarrow \mathrm{e}^{i \phi_{2}}|L\rangle$, the phase modulation of metasurface at each point should satisfy:

$$
\begin{aligned}
& \exp \left(i \phi_{1}(x, y)\right)=\exp \left(i \varphi_{x}(x, y)\right) \exp (i 2 \psi(x, y)) \\
& \exp \left(i \phi_{2}(x, y)\right)=\exp \left(i \varphi_{x}(x, y)\right) \exp (-i 2 \psi(x, y))
\end{aligned}
$$

where $\varphi_{x}(x, y)$ represents the propagation phase, and $\pm i 2 \psi(x, y)$ is the P-B phase. Therefore, we can get $\varphi_{x}(x, y)=\left(\phi_{1}(x, y)+\phi_{2}(x, y)\right) / 2, \quad \psi(x, y)=\left(\phi_{1}(x, y)-\right.$ $\left.\phi_{2}(x, y)\right) / 4$, where $\psi(x, y)$ is the orientation angle of optical axis of structural units. To experimentally realize the CVB multiplexer/demultiplexer, we use the subwavelength $\mathrm{Au}$ nanoantennas with a fixed height of $50 \mathrm{~nm}$ while varied length, width, and rotation angle. As shown in the red dotted frame of Fig. 2a, the proposed metasurface is composed of sandwiched structure. To be specific, from the top to the bottom, they are $\mathrm{Au}$ nanoantennas with thickness of $50 \mathrm{~nm}$, silica film with $200 \mathrm{~nm}$ thickness, gold film with $150 \mathrm{~nm}$ thickness, and silicon wafer. The lattice constant is set to $800 \mathrm{~nm}$, and the length and width of $\mathrm{Au}$ nanoantennas are $l$ and $w$, respectively. The height of nanoantennas and the lattice constant are selected by comprehensively considering the fabrication accuracy of the processing equipment and the optimal performance of the metasurface after sweeping the geometrical parameters of the unit cell. The $\mathrm{Au}$ is chosen as the building material of the metasurface due to its high reflectivity in a broadband from visible to infrared. The demanded propagation phase of $\varphi_{x}$ and $\varphi_{y}$ are shown in Fig. 2c. To meet the half-wave retardance, the phase difference between $\varphi_{x}$ and $\varphi_{y}$ is a constant $\pi$. In this case, the phase delay induced by the P-B phase is given by $\exp ( \pm i 2 \psi(x, y))$. As shown in Fig. 2 d, $\varphi_{1}, \varphi_{2}$, and $\varphi_{3}$ are the phase delay of three metallic nanoantennas with different orientation angles for LHCP beams. This metasurface-based multiplexer/demultiplexer is fabricated on a $\mathrm{SiO}_{2}$ - $\mathrm{Au}$-Si wafer with a $150 \mathrm{~nm}$-thick PMMA layer by standard electron beam lithography technology. 


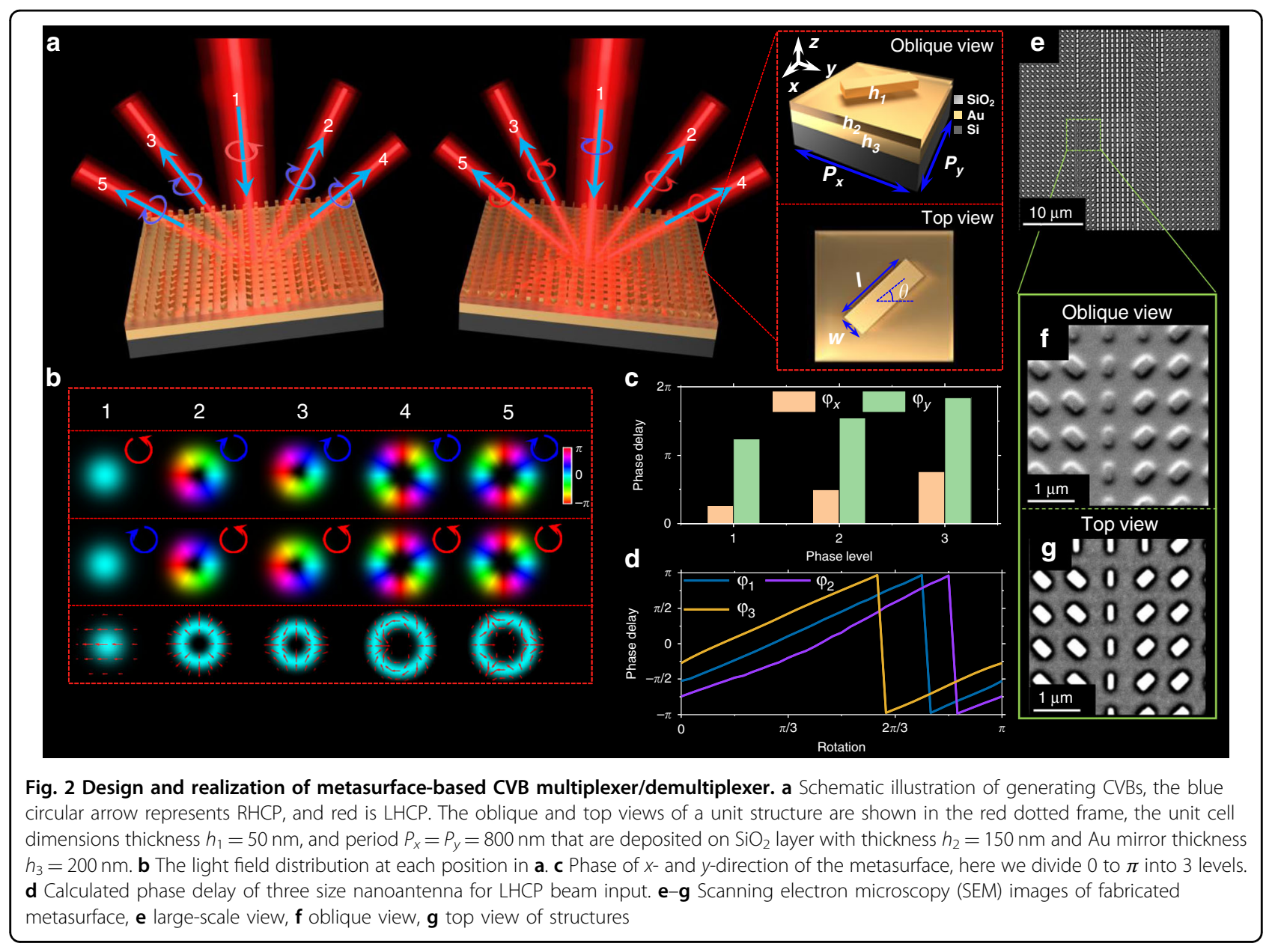

The scanning electron microscopy (SEM) images are shown in Fig. 2e, and the oblique and top view are shown in Fig. 2f, g. The detailed fabrication process is described in the "Materials and methods" section.

\section{Performance of metasurface-based off-axis polarization control}

The characteristics of the produced CVBs have been experimentally investigated, where the experimental setup is schematically shown in Supplementary Note 4 . The far-field intensity patterns of different polarized incident beams are captured by a near-infrared camera, which are shown in Fig. 3a. When a linearly polarized Gaussian beam is incident, the CVBs with the polarization orders from -2 to +2 at corresponding diffraction orders are obtained. As shown in Fig. 3a, the left column shows the theoretical optical intensity and polarization distributions at the diffraction orders of +1 and -2 , where the red arrow represents the polarization direction at the beam cross-section. The measured intensity and polarization distributions are shown in the middle and right columns of Fig. 3a. The polarization distributions are measured by a linear polarizer (LP) with different rotation angles in front of the camera. The black double-headed arrows show the direction of the polarizer's transmission axis. After filtered by LP, the CVB is decomposed into several side lobes. The number of side lobes depends on the polarization order of CVBs (polarization order is half of the number of side lobes), which rotate with the transmission axis of the LP (the polarization order is positive with the side lobe rotating in the same direction as the optical axis, otherwise it is negative). Based on the number and the rotation direction of side lobes, the polarization orders of the CVBs are $m=+1,-2$, which is consistent with theoretical expectations. We also verified the broadband performance of the metasurface. The reflection coefficients for the circularly polarized light beam $R_{\mathrm{LR}}$ and $R_{\mathrm{LL}}$ are simulated with the finite-difference timedomain (FDTD) method, where $R_{\mathrm{LL}}$ and $R_{\mathrm{LR}}$ represent the proportions of LHCP and RHCP in the output beam (when the incident beam is LHCP beam), respectively. As shown in Fig. 3b, the metasurface's working bandwidth is between 1260 and $1675 \mathrm{~nm}$. The scatter plots of Fig. 3b are the measured reflection coefficients of $R_{\mathrm{LR}}$ and $R_{\mathrm{LL}}$ 

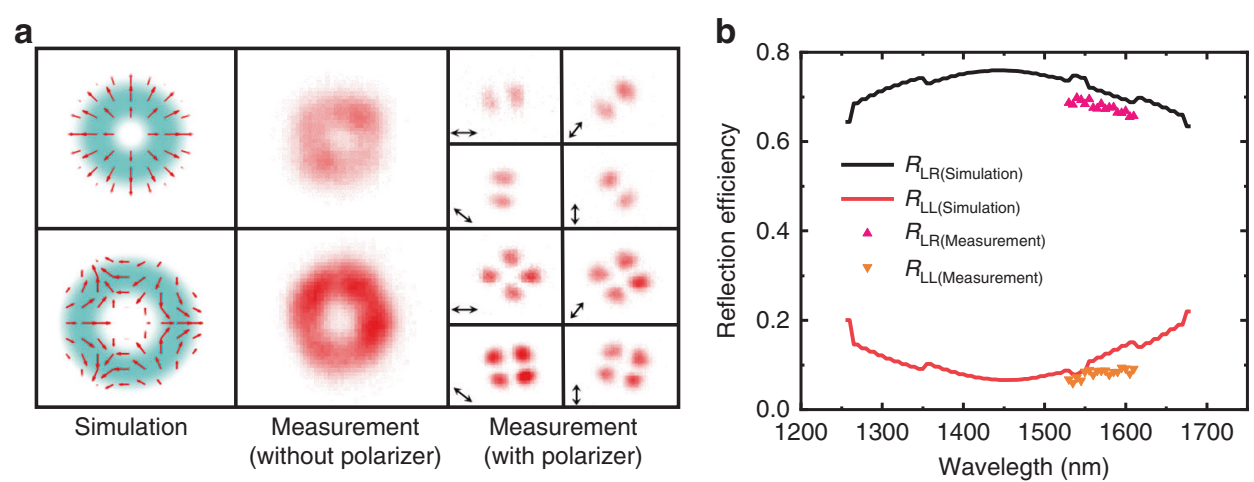

Fig. 3 Optical characterization of the metasurface-based CVB multiplexer/demultiplexer. a Left: simulated optical intensity distribution of the CVB at different diffraction order $(m=+1$ and $m=-2)$; middle and right: measured intensity profiles are shown both with and without a linear polarizer placed in front of the camera. $\mathbf{b}$ Simulated and measured reflection coefficients $R_{\mathrm{LR}}$ and $R_{\mathrm{LL}}$ for circularly polarized light

from 1529 to $1605 \mathrm{~nm}$. The slight disagreement between theoretical and experimental results might arise from the experimental errors, including the metasurface fabrication and optical characterization. Furthermore, we measured the metasurface's far-field distribution at the working wavelength of $1310 \mathrm{~nm}$ (see Supplementary Note 5). The results indicate that the metasurface can also perform CVB multiplexing/demultiplexing at $\lambda=1310 \mathrm{~nm}$.

\section{CVB multiplexing communication}

We employ two metasurfaces as CVB multiplexer and demultiplexer, and the multiplexing/demultiplexing experiment is schematically depicted in Fig. 4. Four Gaussian beams carrying digital signals are incident on the multiplexer at the angles of different diffraction orders (which are labeled as \pm 1 and \pm 2 ). All the incident Gaussian fundamental modes are transformed into CVBs with different polarization orders (dependent on incident angle) and coaxially transmitted along with the zeroth diffraction order. For signal demodulation, according to our previous works ${ }^{40-42}$, the CVB with polarization order $(-m)$ can be recovered to the fundamental spatial mode by using the corresponding metasurface, which can also be used to generate $m$-th CVB. Figure 4a1, a2 shows the schematic illustration of the conversion from CVB to fundamental spatial mode. If a Gaussian beam is incident, the CVBs with the polarization orders from -2 to +2 are obtained at different diffraction orders, and $M$ represents the diffraction order in Fig. 4a. The polarization and intensity distributions of the CVBs with different orders are shown in Fig. 4a1. The beam radius is proportional to the absolute value of its polarization order. If a CVB with $m=-1$ is incident, a Gaussian beam is obtained at the diffraction order of +1 (Fig. 4a2). In addition, the polarization order of CVB in other diffractive order is changed. For example, in the diffraction order of $M=-2$, the polarization order of CVB transforms from $m=-2$ to $m=-3$. Hence, at the receiver, the coaxially transmitted CVBs are demultiplexed into spatially separated Gaussian beams by the metasurface. Figure $4 \mathrm{~b} 1-\mathrm{b} 5$ shows the measured intensity distributions of the multiplexed coaxial beam and demultiplexed CVBs at each diffraction orders. Owing to the vector mode conservation, only the vector mode with inverse polarization order is recovered to fundamental spatial mode with a relatively stronger intensity distribution at the center, which is filtered out by a fundamental mode filter like an aperture. Finally, the recovered fundamental spatial modes are coupled into optical fibers for signal detection.

As a proof of concept, we utilize these metasurfaces to multiplex and demultiplex the CVBs with $m= \pm 1, \pm 2$ (see Supplementary Note 6). For the incident $x$-polarized and $y$-polarized Gaussian beam, the radial and azimuthal CVB can be obtained due to the orthogonality of $x$ - (x-pol) and $y$-direction linear polarization ( $y$-pol). The radial and azimuthal CVB are also orthogonal. Hence, polarizationdivision-multiplexing and mode-division-multiplexing can be combined. We realized four CVB modes multiplexing and demultiplexing at the wavelength of $1554 \mathrm{~nm}$ by combining polarization-division-multiplexing, which carry $400 \mathrm{Gbit} / \mathrm{s}$ quadrature phase shift keying (QPSK) signals. The light intensity matrix of the four demultiplexed CVBs $(m= \pm 1, \pm 2)$, which were applied in the communication system, is shown in Fig. 5a.

The diagonal of the matrix is the intensity of the optical signal obtained by demodulation, and the others are crosstalk. The crosstalk relates to the difference in polarization orders between adjacent channels. Here we set the interval between adjacent channels to $\Delta m=1$, and the crosstalk between adjacent channels can be reduced by increasing $\Delta m$. Hence, the BER performance of this communication system can be further improved. Figure $5 \mathrm{~b}$ represents the BERs of the CVB channels at $1554 \mathrm{~nm}$. " $-2, x$-pol" represents the channel is $x$ - 


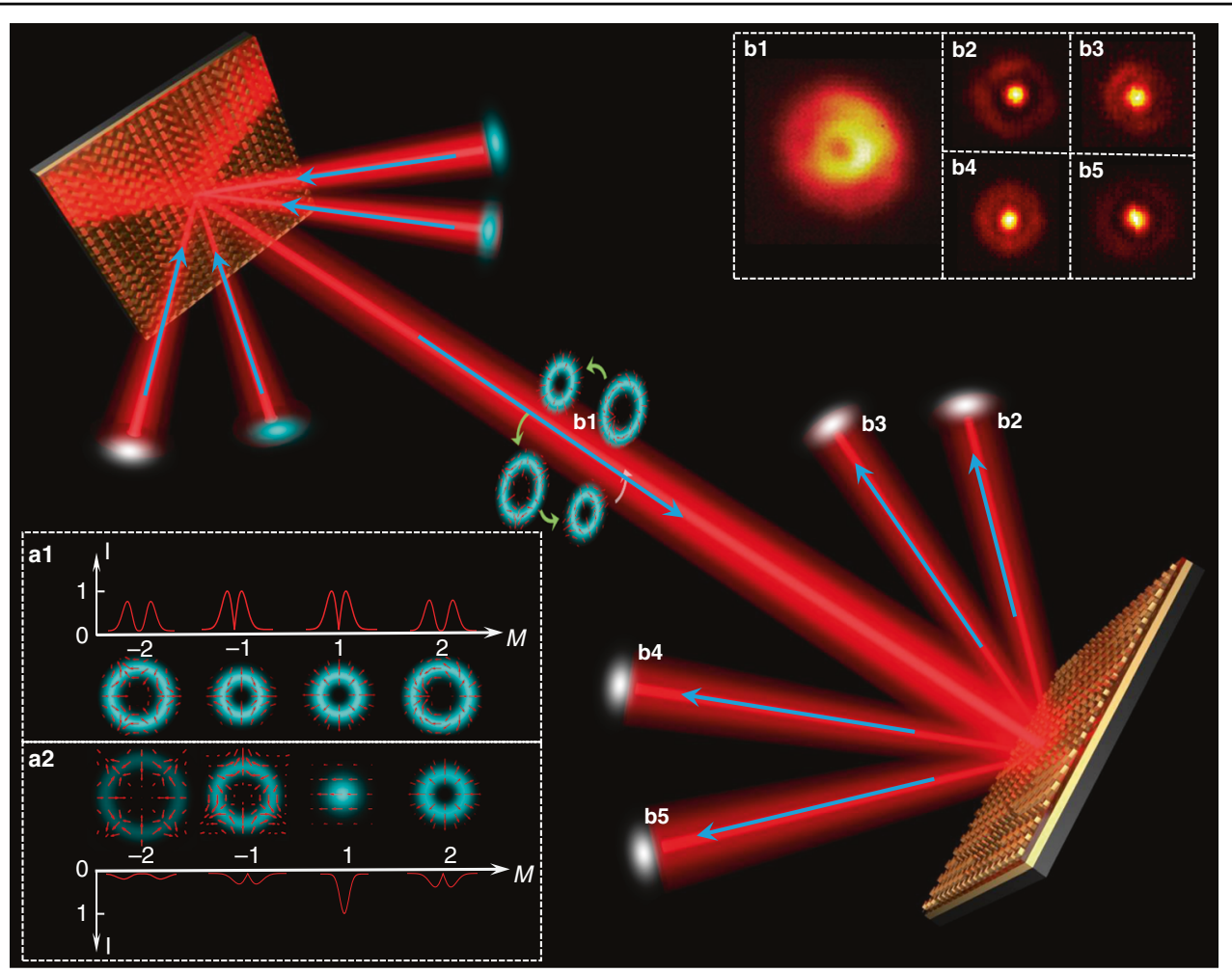

Fig. 4 Schematic of CVB modes multiplexing/demultiplexing by using metasurface-based Dammann optical vector gratings. a1 Polarization and intensity distributions in each diffraction order with Gaussian beam incident, respectively. a2 Polarization and intensity distributions in each diffraction order with CVB $(m=-1)$ incident. b1 Measured coaxial CVB with 4 modes $(m= \pm 1, \pm 2)$; b2 - b5 measured Gaussian points after demultiplexing of the different CVB mode channels

polarization input and polarization order is $m=-2$, and the others can be done similarly. The BERs are all below the hard-decision forward-error-correction (FEC) threshold of $3.8 \times 10^{-3}$. These results help to confirm that the metasurfaces can realize low-crosstalk and high-speed CVB mode multiplexing/demultiplexing.

We further construct a CVB mode multiplexing communication system, which combines wavelength-divisionmultiplexing and polarization-division-multiplexing. The optical spectra at the wavelengths of 1551.72, 1553.33, 1554.94, and $1556.55 \mathrm{~nm}$ are measured before and after multiplexing (see Supplementary Note 7). It can be noticed from Fig. S7 that the linewidth of resonant peaks is broadened as the power of signal is amplified by Erbium-doped fiber amplifier. However, the peak wavelength remains unchanged after propagating through the metasurface, indicating that the metasurface is nondispersive over certain bandwidth. Moreover, we test the BERs of the CVB channels with different vector modes and wavelengths to analyze data signals. The BERs of 32 channels, including 4 wavelengths, 2 polarizations, and $4 \mathrm{CVB}$ modes, are measured.

As shown in Fig. 6a, we select eight channels to analyze the communication performance. " $+1,1556$ " represents the CVB channel at the wavelength of $1556 \mathrm{~nm}$ with $m=+1$, and the rest is done in the same manner. It can be found that the BERs are all below the FEC threshold. When the received power reaches $-19 \mathrm{dBm}$, there is almost no BER. Figure $6 \mathrm{~b}$ depicts the constellations of CVB channels $(m=$ $-2,+1$ ) with the wavelengths of $1551,1553,1554$, and $1556 \mathrm{~nm}$ (at the receiver power of $-22 \mathrm{dBm}$ ). The constellations at 1551,1553,1554, and $1556 \mathrm{~nm}$ are similar at different CVB modes, and the constellations of different CVB modes at the same wavelength are also similar, which demonstrate that the received crosstalk of each channel are equivalent, and the communication system performance is reliable. Furthermore, it should be noted that all channels were individually modulated, detected, and simultaneously analyzed in real time. These results indicate that the metasurface is effective in CVB multiplexing communication.

\section{Discussion}

Coupling and separating CVB modes are two critical procedures in multiplexing/demultiplexing, where off-axis manipulation of CVBs is highly demanded. To control the light beams off-axially, the gradient phase or Dammann vortex grating is usually employed to manipulate the wavevector based on phase modulation ${ }^{46-49}$. However, 

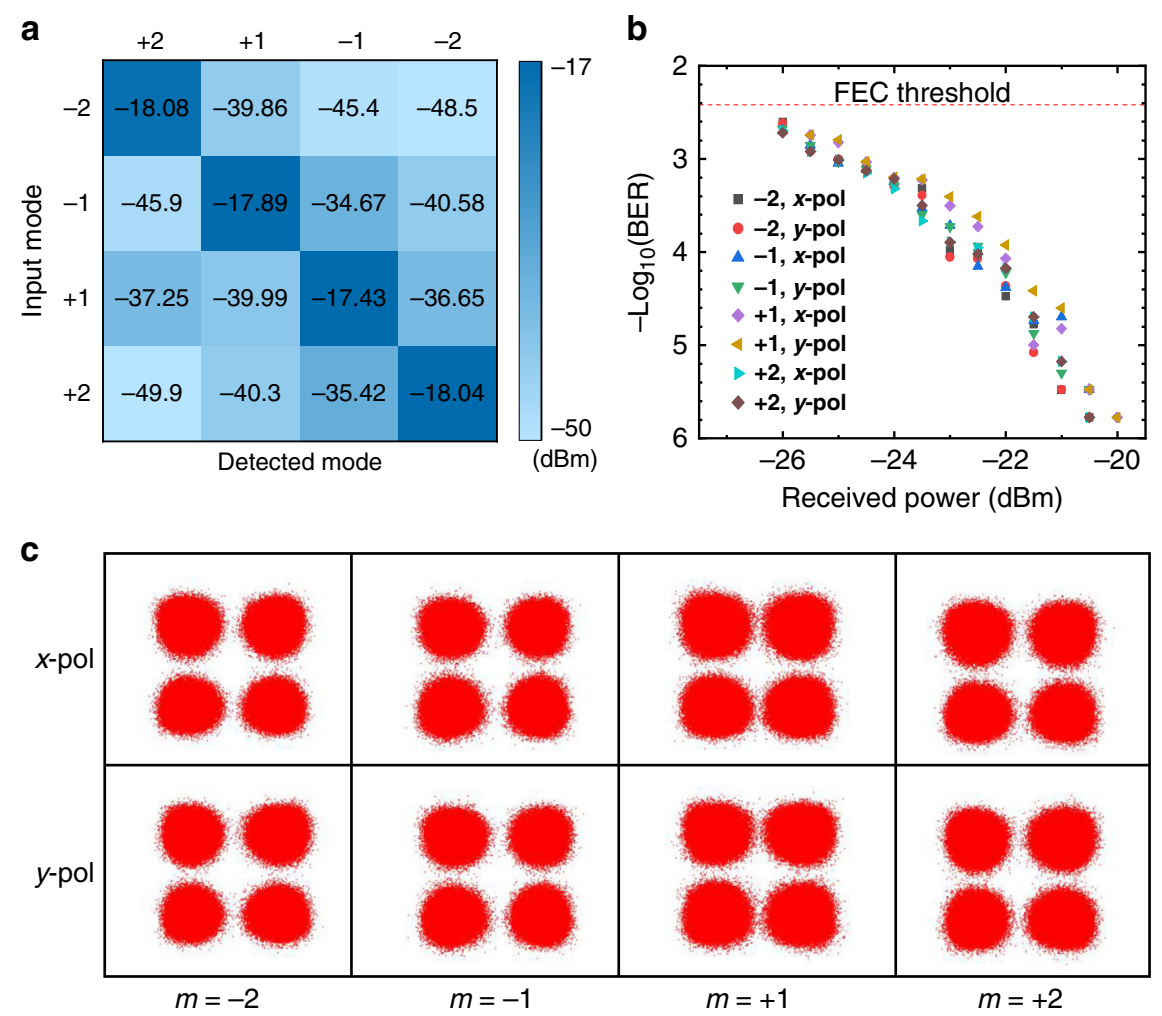

Fig. 5 Experimental results are used to prove that the metasurface-based CVB mode multiplexer/demultiplexer is compatible with polarization multiplexing. a Measured mode crosstalk of four CVB mode channels. b BERs corresponding to eight CVB mode channels at $1554 \mathrm{~nm}$. c Constellations of the QPSK signals of CVB modes $(m= \pm 1, \pm 2)$ with $x$-pol and $y$-pol, respectively
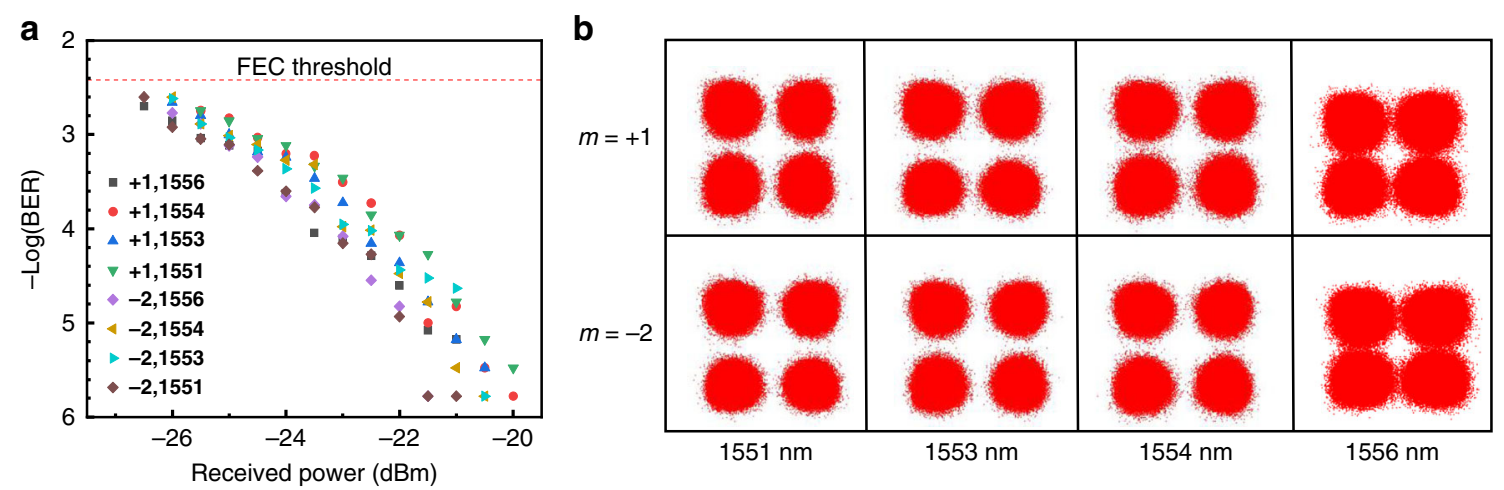

Fig. 6 Demonstration of the CVB mode multiplexer/demultiplexer is compatible with wavelength multiplexing. a Measured BERS of two CVB modes at four wavelengths. b Constellations of QPSK signals carried by CVB modes $(m=-2, m=+1)$ at different wavelengths $(1551$, 1553, 1554, and $1556 \mathrm{~nm}$

these methods are no longer effective for CVBs due to its inhomogeneous polarization states. According to Jones matrix analysis, CVB can be obtained by linearly superposing two orthogonal circularly polarized vortex beams with conjugate topological charges. After decomposing into LHCP and RHCP components, CVBs can be off-axis controlled by independently modulating the phase of these two components, and thus the mode coupling and separating can be achieved via introducing gradient phase changes. Although a spin-multiplexing metasurfacebased Dammann vortex grating can be utilized to measure the phase and polarization singularities of light by using detour phase ${ }^{50}$, the two spin components should be essentially projected into two opposite positions, which is incapable of CVB mode multiplexing/ demultiplexing. 
We independently design the phase modulation for RHCP and LHCP components by exploiting the P-B and propagation phase of metasurface. We adopted the $\mathrm{Au}$ plasmonic metasurface together with the binarized grating structure to off-axially control CVBs. Because the nano-brick needs to satisfy the half-wave condition $\left(\phi_{x}-\right.$ $\left.\phi_{y}=\pi\right)$, the binary grating phase is also a key point for off-axis polarization modulation using plasmonic metasurface. Due to the high reflection efficiency of the reflection-type design and the broadband response characteristics of $\mathrm{Au}$ and P-B phase, these CVB multiplexer/ demultiplexers have high efficiency at $\mathrm{C}$ - and L-band. In addition, owing to the stability of $\mathrm{Au}$ metal, these $\mathrm{CVB}$ multiplexer/demultiplexers can be applied in complex environments, such as high temperature and pressure. According to the principle of optical path reversibility and mode conservation, we simultaneously multiplex and demultiplex CVBs by using the metasurfaces. It is worth mentioning that there are some aspects that could be further improved. For example, it is possible to increase the number of channels while maintaining relatively high conversion efficiency and mode purity by adjusting the structure of the vector grating (see Supplementary Note 8). It is anticipated that this off-axis polarization control method may open a new perspective for CVB applications, such as CVB multiplexing and integrated photonics. Moreover, it can be further applied to CVB holography, particle capture technology, and combine with active metasurfaces to realize dynamic off-axis control.

In summary, we proposed a metasurface-based off-axis polarization control method for CVB multiplexing/demultiplexing. We choose the CVBs with an interval of 1 to verify the feasibility of the communication system and find that the measured BERs are above the FEC threshold in the proposed communication system. Furthermore, the metasurface can be simultaneously used for wavelength-division-multiplexing, polarization-division-multiplexing, and CVB mode-divisionmultiplexing. We achieve a data capacity of $1.56 \mathrm{Tbit} / \mathrm{s}$ $(32 \times 50 \mathrm{Gbit} / \mathrm{s})$ by multiplexing 32 channels (4 wavelengths, 4 CVB modes, and 2 polarizations) and each channel is loaded with $50 \mathrm{Gbit} / \mathrm{s}$ QPSK signals. Since the metasurface is flat and compact, it is highly promising for system integration and miniaturization. Such a technique may find applications in high-capacity communication system.

\section{Materials and methods}

\section{Numerical calculations}

All numerical simulations are performed by using the commercially available software FDTD Solutions (Lumerical Solutions Corp.). In the simulation, a linearly $x$-polarized plane wave (propagated along $z$-direction) with central wavelength of $\lambda=1550 \mathrm{~nm}$ is normally incident onto a single nanorod. In order to design the unit cell of the metasurface, periodic boundary conditions are applied along the $x$ - and $y$-directions, while perfectly matching layer is imposed on the boundary along $z$ direction. The propagation phase $\left(\varphi_{x}\right.$ and $\left.\varphi_{y}\right)$ and power reflection $\left(R_{x}\right.$ and $\left.R_{y}\right)$ (see Supplementary Fig. S9) are obtained by sweeping the geometrical parameters of the nanopillars (width and length varying from 50 to $700 \mathrm{~nm}$ with an interval of $10 \mathrm{~nm}$ ). Moreover, we could retrieve the reflection coefficients for circularly polarized light as $R_{\mathrm{LL}}=\left(R_{x x}+R_{y y}-\left(R_{y x}-R_{x y}\right) \cdot i\right) / 2$ and $R_{\mathrm{LR}}=$ $\left(R_{y y}-R_{x x}-\left(R_{x y}+R_{y x}\right) \cdot i\right) / 2$ from the reflection of linear polarized light, where $R_{x x}$ and $R_{x y}$ represent the proportion of $x$ - and $y$-polarized light, respectively. Here the number $N$ of unit cell for the metasurface is set as $40 \times 40$ and the simulated area along the transverse plane is set as $32 \times 32 \mu \mathrm{m}^{2}$. The simulation results of metasurface at different wavelengths are shown in Fig. S10 of Supplementary Note 9.

\section{Fabrication of the designed metasurface}

The designed metasurfaces consisting of nanoantennas are fabricated based on the standard Electron Beam Lithography (EBL). First, a thin layer of titanium (Ti, thickness: $2 \mathrm{~nm}$ ) is deposited on the silicon substrate, which helps to increase the adhesion of the silicon ( $\mathrm{Si}$ ) substrate. Subsequently, a gold layer (thickness: $200 \mathrm{~nm}$ ) is deposited onto the thin $\mathrm{Ti}$ substrate by using the electron beam evaporator (ASB-EPI-C6). Then, a $\mathrm{SiO}_{2}$ spacer (thickness: $150 \mathrm{~nm}$ ) is deposited onto the gold layer substrate. After that, the positive resist film (polymethylmethacrylate (PMMA), $950 \mathrm{~K}$ ) is spin-coated on the $\mathrm{SiO}_{2}-\mathrm{Au}-\mathrm{Si}$ substrate. In order to obtain a PMMA layer with $150 \mathrm{~nm}$ thickness, the sample is put into the homogenizer with the speed of $4000 \mathrm{rpm}$ for $1 \mathrm{~min}$ and then baked at $180^{\circ} \mathrm{C}$ for $1.5 \mathrm{~min}$. The pattern of the objective nanostructures is etched on the PMMA film by EBL (EBPG 5150) with an accelerating voltage of $100 \mathrm{keV}$ and beam current of $2 \mathrm{nA}$. After etching by EBL, the sample with size of approximately $640 \times 640 \mu \mathrm{m}^{2}$ is put into the developer for $1 \mathrm{~min}$ and then into the fixer for $30 \mathrm{~s}$. The remaining fixer is removed by nitrogen. After that, a $50 \mathrm{~nm}$ gold film is deposited on the sample via thermal evaporation. Finally, the sample is put in a $30 \%$ acetone solution for $6 \mathrm{~h}$, where the excess PMMA and $\mathrm{Au}$ are removed.

\footnotetext{
Acknowledgements

This project was funded by National Natural Science Foundation of China (61805149, 62101334, 12047539, U1701661); Guangdong Basic and Applied Basic Research Foundation (2020A1515011392, 2020A1515110572, 2019A1515111153, 2021A1515011762); Shenzhen Fundamental Research Program (JCYJ20200109144001800, JCYJ20180507182035270); Science and Technology Project of Shenzhen (GJHZ20180928160407303); Shenzhen Universities Stabilization Support Program (SZWD2021013); Shenzhen Excellent Scientific and Technological Innovative Talent Training Program (RCBS20200714114818094); and China Postdoctoral Science Foundation (2020M682867).
} 


\section{Author contributions}

S.C. conducted experiments, analyzed data, and performed theoretical analysis; Z.X. and H.Y. conducted experiments, designed the metasurface, and produced simulation results; X.W. and Y.H. were responsible for communication verification experiments; Z.G. participated in discussions and provided suggestions; Y.L., X.Y., and D.F. supervised the research project. All authors reviewed the manuscript.

\section{Conflict of interest}

The authors declare no competing interests.

Supplementary information The online version contains supplementary material available at https://doi.org/10.1038/s41377-021-00667-7.

Received: 10 June 2021 Revised: 18 October 2021 Accepted: 18 October 2021

Published online: 02 November 2021

\section{References}

1. Richardson, D. J., Fini, J. M. \& Nelson, L. E. Space-division multiplexing in optical fibres. Nat. Photonics 7, 354-362 (2013).

2. Wang, J. et al. Terabit free-space data transmission employing orbital angular momentum multiplexing. Nat. Photonics 6, 488-496 (2012)

3. Luo, L. W. et al. WDM-compatible mode-division multiplexing on a silicon chip. Nat. Commun. 5, 3069 (2014).

4. Zhu, B. et al. 112-Tb/s Space-division multiplexed DWDM transmission with 14-b/s/Hz aggregate spectral efficiency over a 76.8-km seven-core fiber. Opt. Express 19, 16665-16671 (2011).

5. Huang, H. et al. $100 \mathrm{Tbit} / \mathrm{s}$ free-space data link enabled by three-dimensional multiplexing of orbital angular momentum, polarization, and wavelength. Opt. Lett. 39, 197-200 (2014).

6. Zhang, J. W. et al. Mode-division multiplexed transmission of wavelengthdivision multiplexing signals over a 100-km single-span orbital angular momentum fiber. Photonics Res. 8, 1236-1242 (2020).

7. Winzer, P. J. High-spectral-efficiency optical modulation formats. J. Lightwave Technol. 30, 3824-3835 (2012)

8. Chen, Z. Y. et al. Use of polarization freedom beyond polarization-division multiplexing to support high-speed and spectral-efficient data transmission. Light. Sci. Appl. 6, e16207 (2017).

9. Xu, H. X. et al. Spin-encoded wavelength-direction multitasking Janus metasurfaces. Adv. Optical Mater. 9, 2100190 (2021).

10. Deng, L. G. et al. Malus-metasurface-assisted polarization multiplexing. Light. Sci. Appl. 9, 101 (2020)

11. Li, J. Q. et al. Wearable conformal metasurfaces for polarization division multiplexing. Adv. Optical Mater. 8, 2000068 (2020).

12. Wang, J., He, S. L. \& Dai, D. X. On-chip silicon 8-channel hybrid (de)multiplexer enabling simultaneous mode- and polarization-division-multiplexing. Laser Photonics Rev. 8, L18-L22 (2014).

13. Wang, C. F. et al. Cylindrical vector beam multiplexing for radio-over-fiber communication with dielectric metasurfaces. Opt. Express 28, 38666-38681 (2020).

14. He, Y. L. et al. All-optical signal processing in structured light multiplexing with dielectric meta-optics. ACS Photonics 7, 135-146 (2020).

15. Liu, J. et al. Direct fiber vector eigenmode multiplexing transmission seeded by integrated optical vortex emitters. Light. Sci. Appl. 7, 17148 (2018).

16. Nazemosadat, E. et al. Dielectric broadband metasurfaces for fiber modemultiplexed communications. Adv. Optical Mater. 7, 1801679 (2019).

17. Parigi, V. et al. Storage and retrieval of vector beams of light in a multipledegree-of-freedom quantum memory. Nat. Commun. 6, 7706 (2015).

18. Qiao, W. et al. Approach to multiplexing fiber communication with cylindrical vector beams. Opt. Lett. 42, 2579-2582 (2017)

19. Ndagano, B. et al. Fiber propagation of vector modes. Opt. Express 23 17330-17336 (2015)

20. Cheng, W., Haus, J. W. \& Zhan, Q. W. Propagation of vector vortex beams through a turbulent atmosphere. Opt. Express 17, 17829-17836 (2009).
21. Zhu, Z. Y. et al. Compensation-free high-dimensional free-space optical communication using turbulence-resilient vector beams. Nat. Commun. 12 , 1666 (2021).

22. Willner, A. E. Vector-mode multiplexing brings an additional approach for capacity growth in optical fibers. Light. Sci. Appl. 7, 18002 (2018).

23. Shu, W. X. et al. Polarization evolution of vector beams generated by q-plates. Photonics Res. 5, 64-72 (2017).

24. Milione, G. et al. $4 \times 20 \mathrm{Gbit} / \mathrm{s}$ mode division multiplexing over free space using vector modes and a q-plate mode (de)multiplexer. Opt. Lett. 40, 1980-1983 (2015).

25. Yang, Y. J. et al. Optical trapping with structured light: a review. Adv. Photonics 3, 034001 (2021).

26. Milione, G. et al. Higher order pancharatnam-berry phase and the angular momentum of light. Phys. Rev. Lett. 108, 190401 (2012).

27. Forbes, A., Dudley, A. \& McLaren, M. Creation and detection of optical modes with spatial light modulators. Adv. Opt. Photonics 8, 200-227 (2016).

28. Zheng, S. Q. et al. Improve polarization topological order sorting with the diffractive splitting method. Opt. Lett. 44, 795-798 (2019).

29. Fang, J. C. et al. Spin-dependent optical geometric transformation for cylindrical vector beam multiplexing communication. ACS Photonics $\mathbf{5}$ 3478-3484 (2018).

30. Berkhout, G. C. G. et al. Efficient sorting of orbital angular momentum states of light. Phys. Rev. Lett. 105, 153601 (2010)

31. Wen, Y. H. et al. Spiral transformation for high-resolution and efficient sorting of optical vortex modes. Phys. Rev. Lett. 120, 193904 (2018).

32. Wen, $Y ., H$. et al. Compact and high-performance vortex mode sorter for multi-dimensional multiplexed fiber communication systems. Optica 7, 254-262 (2020).

33. Lei, T. et al. Massive individual orbital angular momentum channels for multiplexing enabled by Dammann gratings. Light. Sci. Appl. 4, e257 (2015).

34. Yue, F. Y. et al. Multichannel polarization-controllable superpositions of orbital angular momentum states. Adv. Mater. 29, 1603838 (2017).

35. Li, Y. et al. Orbital angular momentum multiplexing and demultiplexing by a single metasurface. Adv. Optical Mater. 5, 1600502 (2017).

36. Tan, $H$. Y. et al. A free-space orbital angular momentum multiplexing communication system based on a metasurface. Laser Photonics Rev. 13, 1800278 (2019).

37. Arbabi, A. et al. Dielectric metasurfaces for complete control of phase and polarization with subwavelength spatial resolution and high transmission. Nat. Nanotechnol. 10, 937-943 (2015).

38. Wen, D. D. et al. Geometric metasurfaces for ultrathin optical devices. Adv. Optical Mater. 6, 1800348 (2018).

39. Chen, S. M. et al. High-order nonlinear spin-orbit interaction on plasmonic metasurfaces. Nano Lett. 20, 8549-8555 (2020).

40. Mueller, J. P. B. et al. Metasurface polarization optics: independent phase control of arbitrary orthogonal states of polarization. Phys. Rev. Lett. 118 113901 (2017).

41. Guo, Y. H. et al. Spin-decoupled metasurface for simultaneous detection of spin and orbital angular momenta via momentum transformation. Light. Sci. Appl. 10, 63 (2021).

42. Devlin, R. C. et al. Arbitrary spin-to-orbital angular momentum conversion of light. Science 358, 896-901 (2017).

43. Chen, J., Wan, C. H. \& Zhan, Q. W. Vectorial optical fields: recent advances and future prospects. Sci. Bull. 63, 54-74 (2018).

44. Zhan, Q. W. Cylindrical vector beams: from mathematical concepts to applications. Adv. Opt. Photonics 1, 1-57 (2009).

45. $\mathrm{Yi}, \mathrm{X}$. N. et al. Generation of cylindrical vector vortex beams by two cascaded metasurfaces. Opt. Express 22, 17207-17215 (2014).

46. He, Y. L. et al. Controllable photonic spin Hall effect with phase function construction. Photonics Res. 8, 963-971 (2020).

47. Li, S. Q. et al. Efficient optical angular momentum manipulation for compact multiplexing and demultiplexing using a dielectric metasurface. Adv. Optical Mater. 8, 1901666 (2020)

48. Zhang, J. J., Pendry, J. B. \& Luo, Y. Transformation optics from macroscopic to nanoscale regimes: a review. Adv. Photonics 1, 014001 (2019).

49. Sain, B., Meier, C. \& Zentgraf, T. Nonlinear optics in all-dielectric nanoantennas and metasurfaces: a review. Adv. Photonics 1, 024002 (2019).

50. $\mathrm{Fu}, \mathrm{Y} . \mathrm{N}$. et al. Measuring phase and polarization singularities of light using spin-multiplexing metasurfaces. Nanoscale 11, 18303-18310 (2019). 